\title{
RESISTÊNCIA ESTOMÁTICA, TENSÃO DE ÁGUA NO XILEMA E TEOR DE CLOROFILA EM GENÓTIPOS DE GRAVIOLEIRA
}

\author{
Rejane Jurema Mansur Custódio Nogueira ${ }^{1 *}$; Josué Francisco da Silva Jr.2,3 \\ ${ }^{1}$ Depto. de Biologia - UFRPE, Av. Dom Manuel de Medeiros, s/n, Dois Irmãos - CEP: 52171-900 - Recife, PE. \\ ${ }_{3}^{2}$ Empresa Pernambucana de Pesquisa Agropecuária - IPA, C.P. 1022 - CEP: 50761-000 - Recife, PE. \\ ${ }^{3}$ Bolsista Facepe. \\ *Autor correspondente <alisboa@elogica.com.br>
}

RESUMO: As variáveis ecofisiológicas exercem grande influência, não somente no comportamento vegetativo das plantas cultivadas, mas sobretudo no seu desempenho em relação às características produtivas. Este trabalho verificou a resistência à difusão de vapor, as relações hídricas e o teor de clorofila em dois genótipos de gravioleira (Morada e Comum), sob condições naturais de cultivo. O estudo foi realizado num pomar comercial do município de Paudalho, PE, em duas épocas (setembro e dezembro de 1998). O delineamento estatístico foi o inteiramente casualizado com seis repetições para cada genótipo. De cada repetição foram selecionadas duas folhas sadias, completamente expandidas, localizadas no terço médio das copas e diretamente expostas à radiação solar. No horário de maior demanda evaporativa (entre 12 e 14 horas) mediram-se, durante três dias consecutivos para cada época, a transpiração $(E)$, a resistência à difusão de vapor (Rs), a temperatura foliar (Tf), a tensão de água no xilema ( $\Psi w)$ e os teores de clorofila a e $b$. Verificaramse mudanças no comportamento sazonal em relação às trocas gasosas, $\Psi$ w e Tf entre os dois materiais genéticos estudados. Registraram-se correlações entre $\Psi \mathrm{w}$ e $E$ e entre $\Psi \mathrm{w}$ e Tf. A umidade relativa do ar apresentou correlação com a Tf e com a Rs. A gravioleira Comum é mais eficiente fotossinteticamente por minimizar a degradação da clorofila $b$ pela incidência da luz. Os genótipos de gravioleira Morada e Comum apresentaram comportamentos distintos em todos os parâmetros ecofisiológicos avaliados. O Yw constitui um bom parâmetro para aferição de diferenças genotípicas em gravioleira. Já a Rs deve ser utilizada como parâmetro para aferição de adaptação ambiental entre genótipos de gravioleira, em épocas de maior demanda evaporativa.

Palavras-chave: Annona muricata, transpiração, potencial hídrico, resistência difusiva, fotossíntese

\section{STOMATAL RESISTANCE, XYLEM WATER TENSION AND CHLOROPHYLL CONCENTRATION OF SOURSOP GENOTYPES}

\begin{abstract}
Ecophysiological variables have great influence, not only on the vegetative behavior, but mainly in relation to yield characteristics. Leaf diffusive resistence to vapor, xylem water tension and chlorophyll concentration were evaluated for two soursop genotypes (Morada and Comum) cultivated under field conditions. The trial was carried out during two seasons (September and December/1998), in a commercial orchard of Paudalho, PE, Brazil. An entirely randomized experimental design was used, with six replicates per genotype. In each replicate two healthy and completely developed leaves, located on the medial one third of the canopies were selected. These leaves were directly exposed to solar radiation. During three consecutive days on each season, and at the most evaporative activity time (between 12:00 a.m. and 2:00 p.m.), the transpiration (E), leaf diffusive resistance to vapour (Rs), leaf temperature (LT), xylem water tension ( $\Psi w$ ) and the chlorophyll a and $b$ concentrations were measured. Differences in the seasonal behavior were verified in relation to gaseous changes, $\Psi w$ and LT between those genetic materials. Correlations between $\Psi w$ and $E$, and between $\Psi w$ and LT were found. Relative humidity showed correlation with LT and with Rs. Comum soursop was most efficient photosynteticly, decreasing the chlorophyll $b$ degradation by luminosity incidence. In general, genotypes Morada and Comum showed different behavior for all evaluated ecophysiological parameters. $\Psi$ w is a good parameter to distinguish soursop genotypes. Rs can be used as a parameter for differentiation of environmental adaptation among soursop genotypes in higher evaporative requirement seasons.
\end{abstract}

Key words: Annona muricata, transpiration, water potential, diffusive resistance, photosynthesis

\section{INTRODUÇÃO}

As variáveis ecofisiológicas exercem grande influência, não somente no comportamento vegetativo das plantas cultivadas, mas sobretudo no seu desempenho em relação às características produtivas. Os estudos sobre as trocas gasosas e potencial da água da folha em frutíferas, envolvendo a fixação de $\mathrm{CO}_{2}$, transpiração e resistência foliar à difusão de vapor ampliaram-se a partir da década de 80 , em trabalhos direcionados para culturas tropicais, como caju e goiaba (Sena et al., 1995), murici, cupuaçu, biribá, jenipapo e carambola (Oliveira et al., 1995). O comportamento estomático foi avaliado ainda em uva (Kriedemann, 1977; 
Scholefield et al., 1980; Miele, 1989) e especialmente em variedades de citros (Kriedemann, 1968; Rieger, 1992; Machado et al., 1994; Pelacani et al., 1995).

Recentemente, Nogueira (1997), Nogueira \& Moraes (1998) e Nogueira et al. (1998) estudaram o comportamento estomático e a tensão da água no xilema em aceroleiras, e Nogueira et al. (1999a) observaram esses mesmos parâmetros em mangaba cultivada no litoral de Pernambuco. Em relação à diferença entre genótipos de uma mesma espécie, Nogueira et al. (1999b) conseguiram comprovar variabilidade genética para variáveis como transpiração, resistência à difusão de vapor e potencial da água foliar.

Segundo Engel \& Poggiani (1991), a eficiência fotossintética está ligada ao teor de clorofila das plantas, afetando o crescimento e influenciando a adaptabilidade das mesmas aos diversos ambientes. Em presença da luz é constantemente sintetizada e destruída pelo processo da foto-oxidação, sendo que a velocidade de decomposição é diretamente proporcional à intensidade luminosa muito alta, causando dessa forma prejuízos para a fotossíntese. De acordo com Lee (1988), estudos realizados evidenciaram que o teor de clorofila varia muito entre as espécies, assim como entre genótipos de uma mesma espécie.

A gravioleira (Annona muricata L.) é umas das fruteiras tropicais com maior potencial para exploração econômica, e a região Nordeste apresenta condições edafo-climáticas ideais para o seu cultivo. No entanto, algumas características ecofisiológicas dessa cultura necessitam ser avaliadas, visando o seu melhor aproveitamento nos diversos ecossistemas que compõem a região.

O presente trabalho teve por objetivo estudar a diferença entre dois genótipos de gravioleira, com relação à transpiração, resistência à difusão de vapor, temperatura foliar, tensão da água no xilema, além do teor de clorofila durante duas épocas do ano, em condições de campo.

\section{MATERIAL E MÉTODOS}

O experimento foi realizado no pomar comercial Acerolândia, localizado no Município de Paudalho, Zona da Mata Norte de Pernambuco. A área situa-se nas coordenadas $7^{\circ} \mathrm{C} 55^{\prime} 58,3^{\prime \prime}$ Sul e $35^{\circ} \mathrm{C} 08^{\prime} 12,8^{\prime \prime}$ Oeste, a uma altitude média de $70 \mathrm{~m}$. Apresenta clima quente e úmido, com temperatura e precipitação pluvial média anual de $25^{\circ} \mathrm{C}$ e $1702,1 \mathrm{~mm}$, respectivamente, e meses chuvosos de março a julho (FIAM, 1994). O solo é do tipo Podzólico Amarelo álico.

O pomar estudado foi formado em sua maioria por plantas propagadas através de sementes, com uma considerável variabilidade fenotípica. As plantas avaliadas estavam com a idade de três anos e se encontravam na fase de frutificação. O espaçamento utilizado foi o de $6 \mathrm{~m} \times 5 \mathrm{~m}$. As plantas foram conduzidas sob regime de irrigação por gotejamento com freqüência de rega de três vezes por semana (20 L/planta/vez), sendo submetidas a todos os tratos culturais recomendados para a cultura.

Para a consecução deste trabalho foram utilizadas seis plantas de cada genótipo de gravioleira (Morada e Comum), avaliadas em duas épocas (setembro e dezembro de 1998). O delineamento estatístico utilizado foi o inteiramente casualizado com seis repetições.

De cada planta foram selecionadas duas folhas sadias e completamente expandidas, localizadas no terço médio das copas e diretamente expostas à radiação solar. No horário de maior demanda evaporativa (entre 12 e 14 horas) mediram-se, durante três dias consecutivos para cada época, a transpiração (E), a resistência foliar à difusão de vapor (Rs) e a temperatura foliar (Tf). Para a obtenção dos referidos dados foi utilizado um porômetro de equilíbrio dinâmico da LICOR, modelo LI-1600. Paralelamente, na câmara do porômetro foram monitoradas a temperatura do ar, a umidade relativa de ar, e, com o auxílio de um sensor quântico, foi medida a radiação fotossinteticamente ativa (PAR). Calculou-se também o déficit de pressão de vapor do ar (DPV) segundo Vianello \& Alves (1991).

O potencial da água da folha $(\Psi w)$ foi determinado utilizando-se uma câmara de pressão de Scholander (Scholander et al., 1965), modelo 3035 da "Soil Moisture Equipement Corp", Santa Bárbara, Califórnia (EUA). Para essas avaliações, foram utilizadas as folhas nas quais foram realizadas as medidas porométricas. As mesmas foram envoltas em filme de PVC antes de destacadas, para evitar desidratação, e postas em caixa de isopor com gelo. Posteriormente, foram colocadas no cilindro da câmara, com a taxa de aumento de entrada de gás mantida constante para todas as medidas.

A extração da clorofila foi realizada em acetona a $80 \%$, utilizando-se cerca de $200 \mathrm{mg}$ de matéria fresca de folhas maduras expostas ao sol, coletadas da parte mediana das plantas, no mês de dezembro, de acordo com o método descrito por Harbone (1984) modificado. A quantificação dos teores de clorofila $a$ e $b\left(\mathrm{mg} \mathrm{L}^{-1}\right)$ foi procedida por espectrofotometria de emissão a 645 e $663 \mathrm{~nm}$, através das equações dadas a seguir, onde A é a absorbância no comprimento de onda utilizado. Os resultados obtidos foram expressos em miligramas de clorofila por grama de matéria fresca.

$$
\begin{aligned}
& \text { Clorofila } a=12,21 \times \mathrm{A}_{663}-2,81 \times \mathrm{A}_{645} \\
& \text { Clorofila } b=20,13 \times \mathrm{A}_{645}+7,18 \times \mathrm{A}_{663}
\end{aligned}
$$

Para análise comparativa entre as médias, utilizou-se ANOVA e, para separação, o teste de Tukey, além da determinação de correlação linear simples, para estimar o grau de relação entre os pares de variáveis. 


\section{RESULTADOS E DISCUSSÃO}

A análise de variância revelou efeito significativo a $5 \%$, para genótipos e épocas de avaliação, em todas as variáveis estudadas. Com relação às interações, a significância não foi observada apenas para o $\Psi \mathrm{w}$ da folha (TABELA 1).

A TABELA 2 mostra as variações do PAR, UR e Tar, mensuradas no interior da câmara do porômetro, durante o período das avaliações. O PAR variou de 1440,0 a $1726,5 \mu \mathrm{mol} \mathrm{m} \mathrm{m}^{-2} \mathrm{~s}^{-1}$, a UR de 42,8 a $53,2 \%$, enquanto que os valores médios da Tar situaram-se entre 32,3 a $36,4^{\circ} \mathrm{C}$.

Constataram-se mudanças no comportamento sazonal das trocas gasosas, de $\Psi$ w e de Tf nos dois genótipos estudados (TABELA 3). De modo geral, os valores da $E$ foram mais elevados em setembro do que em dezembro, sendo que em ambas as épocas o genótipo Morada apresentou valores superiores $(8,03 \mathrm{e}$ $7,95 \mathrm{mmol} \mathrm{m}^{-2} \mathrm{~s}^{-1}$, respectivamente) quando comparados àqueles registrados na Comum $\left(6,43\right.$ e $3,27 \mathrm{mmol} \mathrm{m}^{-2} \mathrm{~s}^{-}$ 1 , respectivamente). Verificou-se em dezembro uma redução na taxa transpiracional dos genótipos da ordem de $1 \%$ e $49 \%$ para Morada e Comum, respectivamente. Como os genótipos foram submetidos às mesmas condições de solo e irrigação, esse comportamento estomático sugere que a Morada seja mais adaptada às condições edafo-climáticas da região. O bloqueio do fluxo de vapor de água retratado pela redução da abertura estomática da graviola Comum, limita a assimilação de $\mathrm{CO}_{2}$, reduzindo dessa forma o crescimento. Tal fato foi observado por Souza et al. (2000) nesse mesmo genótipo submetido a estresse hídrico. Os referidos autores verificaram diminuição da matéria seca nos tratamentos equivalentes a 50 e $25 \%$ da capacidade de campo, sendo esse efeito mais pronunciado na parte aérea que nas raízes. Variações no grau de abertura estomática em matrizes de aceroleira cultivadas nas mesmas condições que as deste experimento, foram observadas por Nogueira (1997).

Os valores médios da Rs em setembro situaramse entre 106,50 s m (Morada) e 131,17 s m$^{-1}$ (Comum). Entretanto, em dezembro essa diferença se acentuou, tornando-se significativa, com variações de $169,33 \mathrm{~s} \mathrm{~m}^{-1}$ a $352,67 \mathrm{~s} \mathrm{~m}^{-1}$, para Morada e Comum, respectivamente. O aumento da Rs nas horas de maior demanda evaporativa reflete mudanças na abertura estomática, reduzindo a absorção de $\mathrm{CO}_{2}$ e, consequentemente, diminuindo a atividade fotossintética. Sena et al. (1995), estudando variações na Rs em diversas culturas perenes, como guaraná, caju e goiaba, nos períodos de seca e de chuvas, verificaram que nesse último a diferença da Rs entre as espécies foi menos evidente, variando de 15 a $30 \mathrm{~s} \mathrm{~m}^{-1}$, enquanto no período de seca essa variação foi muito mais acentuada $\left(250\right.$ a $\left.300 \mathrm{~s} \mathrm{~m}^{-1}\right)$.

TABELA 1 - Síntese da análise de variância para as variáveis fisiológicas estudadas referentes a dois genótipos de gravioleira, cultivados em condições de campo. Paudalho, PE, 1998.

Quadrado Médio

\begin{tabular}{|c|c|c|c|c|c|}
\hline \multirow[b]{2}{*}{ Fonte de Variação } & \multirow[b]{2}{*}{ Grau de Liberdade } & \multicolumn{4}{|c|}{ Quadrado Médio } \\
\hline & & E & Rs & Tf & $\Psi w$ \\
\hline & & 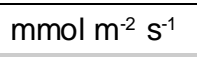 & $\mathrm{s} \mathrm{m}^{-1}$ & ${ }^{\circ} \mathrm{C}$ & $\mathrm{MPa}$ \\
\hline Época (E) & 1 & $15,79^{* *}$ & $12,15^{\star *}$ & $57,04^{\star *}$ & $0,16^{* *}$ \\
\hline Ge nótipo (G) & 1 & $59,17^{\star *}$ & $6,49^{\star \star}$ & $9,63^{\star \star}$ & $0,67^{\star *}$ \\
\hline$E \times G$ & 1 & $14,20^{\star *}$ & $3,76^{\star *}$ & $6,20^{* *}$ & $0,02^{\text {ns }}$ \\
\hline Resíduo & 20 & 0,41 & 0,08 & 0,06 & 0,00 \\
\hline Média Geral & & 6,42 & 189,92 & 34,55 & $-1,64$ \\
\hline CV (\%) & & 10,00 & 14,84 & 0,71 & 4,06 \\
\hline
\end{tabular}

*Significativo pelo teste $\mathrm{F}(\mathrm{P}<1 \%)$; ${ }^{* *}$ Significativo pelo teste $\mathrm{F}(\mathrm{P}<5 \%)$; E - transpiração; Rs - resistência difusiva; Tf - temperatura foliar; $\Psi \mathrm{w}$ - potencial da água da folha.

TABELA 2 - Valores médios e erros-padrão das condições ambientais no interior da câmara do porômetro (Tar e UR), e no plano de cada folha amostrada (PAR) por ocasião das avaliações porométricas e do potencial da água, em gravioleiras cultivadas sob condições de campo.

\begin{tabular}{lcccccc}
\hline \multirow{2}{*}{ Genótipo } & \multicolumn{6}{c}{ Época de avaliação/ Variável climática } \\
\cline { 2 - 7 } & PAR & Tar & UR & PAR & Tar & UR \\
\hline & $\mu \mathrm{mol} \mathrm{m} \mathrm{m}^{-2} \mathrm{~s}^{-1}$ & ${ }^{\circ} \mathrm{C}$ & $\%$ & $\mu \mathrm{mol} \mathrm{m}^{-2} \mathrm{~s}^{-1}$ & ${ }^{\circ} \mathrm{C}$ & $\%$ \\
Comum & $1678,17 \pm 25,38$ & $32,3 \pm 0,15$ & $52,90 \pm 0,07$ & $1440,00 \pm 20,81$ & $35,27 \pm 0,08$ & $42,83 \pm 0,03$ \\
Morada & $1698,33 \pm 21,82$ & $32,5 \pm 0,08$ & $53,2 \pm 0,00$ & $1726,50 \pm 15,57$ & $36,43 \pm 0,13$ & $43,20 \pm 0,00$ \\
\hline
\end{tabular}

Scientia Agricola, v.58, n.3, p.491-495, jul./set. 2001 
Os genótipos estudados apresentaram Tf superior às da câmara do porômetro, exceto a graviola Comum, no mês de dezembro $\left(34,95^{\circ} \mathrm{C}\right)$. Por outro lado, nesse mesmo mês, a Morada apesar de apresentar maiores taxas transpiracionais, mostrou uma Tf superior a $2^{\circ} \mathrm{C}$ em relação à Comum. É possível que esses resultados tenham sido influenciados pela variação da radiação solar incidida no plano da folha, no momento das medições. Têm sido registradas na literatura $\mathrm{Tf}$ superiores às do ambiente, em plantas submetidas a estresses sazonais. Diferenças nas $\mathrm{Tf}$ de até $4^{\circ} \mathrm{C}$ acima da temperatura do ambiente foram observadas em aceroleiras de oito meses de idade cultivadas na Zona da Mata de Pernambuco, durante as horas de maior demanda evaporativa (Nogueira \& Moraes, 1998).

As médias do $\Psi w$ foliar obtidas para os genótipos Morada e Comum no mês de setembro, foram de $-1,69$ $\mathrm{MPa}$ e -1,42 MPa, respectivamente. Esses valores declinaram para -1,91 $\mathrm{MPa}$ e $-1,52 \mathrm{MPa}$, respectivamente, no mês de dezembro. Os valores mais negativos do $\Psi \mathrm{w}$ constatados no genótipo Morada, provavelmente decorrem das maiores taxas transpiratórias apresentadas, uma vez que o solo mostrou-se com boa disponibilidade de água para as raízes. Comparando-se o $\Psi w$ encontrado neste trabalho com os de aceroleiras cultivadas no semi-árido por Nogueira et al. (1998), verifica-se que estão acima das faixas encontradas nas horas mais quentes do dia $(-3,26 \mathrm{MPa})$.

Na TABELA 4 são apresentadas os coeficientes de correlação simples, onde levaram-se em consideração todas as observações obtidas para cada variável de forma generalizada, ou seja, os tratamentos (genótipos e épocas) não foram isolados. O potencial da água da folha apresentou uma correlação significativa e inversa com a transpiração $\left(r=-0,58^{\star}\right)$ e temperatura foliar $\left(r=-0,71^{*}\right)$. A umidade relativa do ar apresentou uma correlação forte e inversa com a temperatura foliar $\left(r=-0,86^{*}\right)$ e demonstra correlacionar-se também com a resistência difusiva $(r$ $\left.=-0,73^{*}\right)$. O PAR apresentou uma correlação forte $\mathrm{e}$ positiva com a transpiração $\left(r=0,89^{*}\right)$ e inversa com a resistência difusiva $\left(r=-0,87^{\star}\right)$.

$\mathrm{Na}$ Tabela 5 podem ser visualizados os teores de clorofila $a$ e $b$, havendo diferença significativa entre os genótipos apenas para os teores de clorofila $b(0,57$ e $0,43 \mathrm{mg} \mathrm{L}^{-1}$, respectivamente para Comum e Morada). De acordo com Lee (1988), estudos realizados evidenciaram que os teores de clorofila variam muito entre as espécies, assim como entre genótipos de uma mesma espécie. Os resultados obtidos neste trabalho demonstram que a degradação da clorofila $b$ foi menos acentuada na graviola Comum que na Morada.

TABELA 3 - Valores médios de temperatura foliar (Tf), transpiração (E), resistência difusiva (Rs) e potencial da água da folha $(\Psi w)$ em dois genótipos de gravioleira cultivados sob condições de campo em duas épocas do ano. Paudalho, PE, 1998.

\begin{tabular}{|c|c|c|c|c|c|c|c|c|}
\hline \multirow{2}{*}{ Época } & \multicolumn{2}{|c|}{ Tf } & \multicolumn{2}{|c|}{$E$} & \multicolumn{2}{|c|}{ Rs } & \multicolumn{2}{|c|}{$\Psi \mathrm{w}$} \\
\hline & Morada & Comum & Morada & Comum & Morada & Comum & Morada & Comum \\
\hline & \multicolumn{2}{|c|}{${ }^{\circ} \mathrm{C}$} & \multicolumn{2}{|c|}{$\mu \mathrm{mol} \mathrm{m}^{-2} \mathrm{~s}^{-1}$} & \multicolumn{2}{|c|}{$\mathrm{s} \mathrm{m}^{-1}$} & \multicolumn{2}{|c|}{$\mathrm{MPa}$} \\
\hline Setembro & $33,13 \mathrm{bA}$ & $32,88 \mathrm{bA}$ & $8,03 \mathrm{aA}$ & $6,43 \mathrm{aB}$ & $106,50 \mathrm{bA}$ & $131,17 \mathrm{bA}$ & $-1,69 a B$ & $-1,42$ aA \\
\hline Dezembro & $37,23 \mathrm{aA}$ & $34,95 \mathrm{aB}$ & 7,95 aA & $3,27 \mathrm{bB}$ & $169,33 \mathrm{aB}$ & 352,67 aA & $-1,91 b B$ & $-1,52 b A$ \\
\hline
\end{tabular}

Médias seguidas pela mesma letra minúscula na mesma coluna e maiúscula na mesma linha, não diferem significativamente pelo teste de Tukey a $5 \%$.

TABELA 4 - Coeficientes de correlação simples entre as variáveis utilizadas - Temperatura do ar (Tar), temperatura foliar (Tf), Umidade relativa do ar (UR), Radiação fotossinteticamente ativa (PAR), Resistência difusiva (Rs), Transpiração $(E)$, Potencial da água da folha $(\psi w)$ e Déficit de pressão de vapor (DPV).

\begin{tabular}{|c|c|c|c|c|c|c|c|}
\hline Variável & Tar & $\mathrm{Tf}$ & UR & PAR & Rs & $E$ & $\Psi w$ \\
\hline $\mathrm{Tf}$ & $0,99^{*}$ & & & & & & \\
\hline UR & & $-0,86^{\star}$ & & & & & \\
\hline PAR & & & $0,44^{* *}$ & & & & \\
\hline Rs & $0,3^{3 \mathrm{NS}}$ & $0,33^{\mathrm{ns}}$ & $-0,73^{*}$ & $-0,87^{*}$ & & & \\
\hline$E$ & $0,01^{\mathrm{ns}}$ & $0,04^{\mathrm{ns}}$ & $0,43^{* *}$ & $0,89^{*}$ & & & \\
\hline$\Psi w$ & $-0,69^{*}$ & $-0,71^{*}$ & $0,38^{\text {ns }}$ & $-0,46^{* *}$ & $0,24^{\text {ns }}$ & $-0,58^{*}$ & \\
\hline DPV & & $0,97^{*}$ & & & $0,52^{*}$ & $-0,18^{n s}$ & $-0,57^{*}$ \\
\hline
\end{tabular}

*significativo a $5 \%$; ** significativo a $1 \%$; n.s. não-significativo 
TABELA 5 - Teores de clorofilas $a$ e $b$ em dois genótipos de gravioleira cultivados sob condições de campo. Paudalho, PE, 1998.

\begin{tabular}{lcc}
\hline \multirow{2}{*}{ Genótipo } & \multicolumn{2}{c}{ Teor de clorofila } \\
\cline { 2 - 3 } & Clorofila a & Clorofila b \\
\hline Morada & 1,42 a & $0,43 \mathrm{~b}$ \\
Comum & $1,24 \mathrm{a}$ & $0,57 \mathrm{a}$ \\
\hline
\end{tabular}

Médias seguidas pela mesma letra na mesma coluna não diferem significativamente pelo teste de Tukey a $5 \%$.

\section{CONCLUSÕES}

Há influência da sazonalidade no comportamento fisiológico com diferenças genotípicas relacionadas à adaptação ambiental.

Os genótipos de gravioleira Morada e Comum apresentaram comportamentos distintos em todos os parâmetros ecofisiológicos avaliados na época de maior demanda evaporativa.

O potencial da água da folha constitui um bom parâmetro para aferição de diferenças genotípicas em gravioleira.

A resistência à difusão de vapor deve ser utilizada como parâmetro para aferição de adaptação ambiental entre genótipos de gravioleira, em épocas de maior demanda evaporativa.

A gravioleira Comum é mais eficiente fotossinteticamente por minimizar a degradação da clorofila $b$ pela incidência da luz.

\section{REFERÊNCIAS BIBLIOGRÁFICAS}

ENGEL, V.L.; POGGIANI, F. Estudo da concentração de clorofila nas folhas e seu espectro de absorção de luz em função do sombreamento em mudas de quatro espécies florestais nativas. Revista Brasileira de Fisiologia Vegetal, v.3, p.39$45,1991$.

Fundação de Desenvolvimento Municipal do Interior de Pernambuco. Perfil municipal do interior de Pernambuco. Recife: FIAM, 1994. p.639-642.

HARBONE, J.B. Phytochemical methods: a guide to modern techniques of plant analysis. London: Chapman \& Hall, 1984. $216 p$.

KRIEDEMANN, P.E. Some photosynthetic characteristics of citrus leaves. Australian Journal of Biological Sciences, v.21, p.895-905, 1968.

KRIEDEMANN, P.E. Vineleaf photosynthesis. In: INTERNATIONAL SYMPOSIUM ON THE QUALITY OF THE VINTAGE, Cape Town, 1977. Proceedings. Cape Town: South African Minister of Agriculture, Office International de la Vigne et du Vin, 1977.

LEE, D.W. Simulating forest shade to study the development ecology of tropical plants: juvenile growth in three vines in India. Journal of Tropical Ecology, v.4, p.281-292, 1988.

MACHADO, E.C.; QUAGGIO, J.A.; LAGÔA, A.M.M.A.; TICELLI, M.; FURLANI, P.R. Trocas gasosas e relações hídricas em laranjeiras com clorose variegada dos citros. Revista Brasileira de Fisiologia Vegetal, v.6, p.53-57, 1994.

MIELE, A. Influência do sistema de condução na evolução dos açúcares redutores e da acidez total durante a maturação da uva: relação com área foliar, radiação solar e fotossíntese. Revista Brasileira de Fisiologia Vegetal, v.1, p.32-40, 1989.
NOGUEIRA, R.J.M.C. Expressões fisiológicas da aceroleira (Malpighia emarginata D.C.) em condições adversas. São Carlos, 1997. 207p. Tese (Doutorado) - Universidade Federal de São Carlos.

NOGUEIRA, R.J.M.C.; MORAES, J.A.P.V. Ecofisiologia da acerola. I. Transpiração, resistência difusiva e temperatura foliar. In: SEMINÁRIO REGIONAL DE ECOLOGIA, 8., 1996. Anais. São Carlos: Programa de Ecologia e Recursos Naturais, UFSCar, 1998. v.3, p.1085-1091.

NOGUEIRA, R.J.M.C.; BURITY, H.A.; MORAES, J.A.P.V. Transpiração e potencial hídrico foliar em aceroleiras (Malpighia emarginata D.C.) cultivadas na zona semi-árida de Pernambuco. Revista Científica Rural, v.3, p.75-81, 1998. NOGUEIRA, R.J.M.C.; MELO FILHO, P. da A.; ARAÚJO, E. de L. Expressões ecofisiológicas de germoplasma de Hancornia speciosa Gomes cultivado no litoral de Pernambuco. Ciência Rural, v.29, 1999a. /No Prelo/

NOGUEIRA, R.J.M.C.; SILVA JR., J.F. da; BEZERRA, J.E.F.; LEDERMAN, I.E.; BURITY, H.A. Comportamento estomático e tensão de água no xilema em pitangueiras (Eugenia uniflora L.) cultivadas sob estresse hídrico. In: CONGRESSO BRASILEIRO DE FISIOLOGIA VEGETAL, 7., Brasília, 1999. Resumos. Londrina: Sociedade Brasileira de Fisiologia Vegetal, 1999b. p.119.

OLIVEIRA, M.N.S. de; OLIVEIRA, L.E.M.; SOARES, A.M. Comportamento de plantas jovens de algumas espécies frutíferas tropicais e subtropicais. In: CONGRESSO BRASILEIRO DE FISIOLOGIA VEGETAL, 5., 1995 Resumos. Lavras: Sociedade Brasileira de Fisiologia Vegetal, 1995. p.309.

PELACANI, C.R.; CRUZ, J.L.; SOARES FILHO, W.S. Avaliação preliminar de híbridos de citrus para tolerância ao estresse hídrico. In: CONGRESSO BRASILEIRO DE FISIOLOGIA VEGETAL, 5., 1995. Resumos. Lavras: Sociedade Brasileira de Fisiologia Vegetal, 1995. p.389.

RIEGER, M. Growth, gas exchange, water uptake and drought response of seedling-and cutting-propagated peach and citrus rootstocks. Journal of the American Society for Horticultural Science, v.117, p.834-840, 1992.

SCHOLANDER, P.F.; HAMMEL, H.T.; HEMINGSEN, E.A.; BRADSTREET, E.D. Hydrostatic pressure and osmotic potencials in leaves of mangroves and some other plants. Proceedings of the National Academy Science, v.51, p.119$125,1965$.

SCHOLEFIELD, P.B.; NEALES, T.F.; MAY, P. Carbon balance of the Sultana vine (Vitis vinifera L.) and the effects of autumn defoliation by harvest-prunning. Australian Journal of Plant Physiology, v.5, p.561-570, 1980.

SENA, J.O.A.; MEDINA, C.L.; VIRGENS FILHO, A.C.; CASTRO, P.R.C. Variações na transpiração e na resistência estomática de culturas perenes tropicais sob condições de disponibilidade e deficit de água. In: CONGRESSO BRASILEIRO DE FISIOLOGIA VEGETAL, 5., 1995. Resumos. Lavras: Sociedade Brasileira de Fisiologia Vegetal, 1995. p.351.

SOUZA, L.M. de; NOGUEIRA, R.J.M.C.; AZEVEDO NETO, A.D. de. Efeito do estresse hídrico sobre o crescimento da gravioleira (Annona muricata L.) cultivada em casa de vegetação. (Compact disc). In: CONGRESSO BRASILEIRO DE FRUTICULTURA, 16., 2000. Resumos. Fortaleza: Sociedade Brasileira de Fruticultura/ Embrapa Agroindústria Tropical, 2000. p.317.

VIANELLO, R.L.; ALVES, A.R. Meteorologia básica e aplicações. Viçosa: UFV, Imprensa Universitária, 1991. 449p.

Recebido em 26.07.00 